\title{
Results of Magnetic Axis Measurements on a Prototype Main Lattice Quadrupole for the LHC
}

\author{
N. Smirnov, L. Bottura, M. Buzio, G. Deferne, V. Parma, P. Rohmig, and T. Tortschanoff
}

\begin{abstract}
More than 470 twin aperture lattice quadrupoles are needed for the Large Hadron Collider (LHC) under construction at CERN. The lattice quadrupole, assembled with correction magnets in its helium enclosure-the cold mass and integrated in a common cryostat called the Short Straight Section (SSS). All SSS cold mass prototypes have been developed and built by CEA (Saclay) in collaboration with CNRS (Orsay, France). The last SSS prototype (SSS5) was used to investigate the behavior of the magnetic axis through various steps of the installation cycle for the series quadrupoles: including transportation, thermal-cycles, and being lowered into the tunnel. Results of extensive measurements before and after each of these stages are presented here, showing that the effect of transport is weak and within the window of measurement resolution. Also shown is that the long-term stability observed during two years is comparable with the requirements from magnet tolerances. To minimize systematic errors, all tests were performed with two independent measurement systems: Single-Stretched Wire (SSW) and Automated Scanner. A brief description of these systems is given, concentrating mostly on their accuracies.

In addition, establishment of a reliable cold-warm correlation for the magnetic axis position is a very important issue because it can reduce the percentage of cold tests. Though this correlation will be based on proper statistics at the beginning of series production, magnetic axis behavior during thermal-cycle has been investigated for the SSS5 prototype, and these results are also presented.
\end{abstract}

Index Terms-LHC quadrupole, magnetic axis, magnetic measurements.

\section{INTRODUCTION}

$\mathbf{T}$ HE last prototype of the Short Straight Section (SSS5) [1] containing only the superconducting main quadrupole (MQ) has been measured at both the room and $1.9 \mathrm{~K}$ temperatures using the Single Stretched Wire system (SSW) [2] and Automated Scanner [3]. The main objective of those tests was to identify the influence of magnet transportation, thermal cycle and lowering into the tunnel on the magnetic axis position. Therefore each test was split in two measurements: before and after the action. Then the results obtained have been analyzed and compared.

The direct comparison between these two measurement systems was done once at warm temperature. The revealed discrepancies between the magnetic axes measured with these two systems were quite small and acceptable. The difference obtained could be attributed among others to different measurement conditions: AC in case of the SSW and DC in the case of the Scanner.

Manuscript received October 20, 2003.

The authors are with CERN, LHC Division, CH-1211 Geneva 23, Switzerland (e-mail: Nikolay.smirnov@cern.ch).

Digital Object Identifier 10.1109/TASC.2004.829060

\section{Main Measurement Systems fOR AXIS MeASUREMENTS}

Both systems described here are dedicated to axis measurements of the various types of SSS's.

\section{A. Automated Scanner}

This measurement system was already described in detail elsewhere. Here we recall briefly the performance of the system in sense of the axis measurement.

The standard deviation of the axis measurement is approximately $20 \mu \mathrm{m}$ in the reference frame of the alignment telescope and $60 \mu \mathrm{m}$ in the SSS fiducial frame. This value is safely within the target tolerance.

The systematic errors of the system will be discussed in Section II-C.

\section{B. Single Stretched Wire (SSW)}

1) System Description: The second considered system is a single stretched wire (SSW) system similar to that used at DESY for the HERA magnet measurements. The system has been originally developed to measure the integral strength $\left(\int \mathrm{Gdl}\right)$ and centering parameters (magnetic center and roll angle) for the Fermilab Main Injector (FMI) quadrupoles at Magnet Test Facility (MTF). Within the framework of LHC-US collaboration one SSW system was built and delivered to CERN in 2000 and a second system in 2003.

Measurements with the SSW system consist in moving a single wire stretched through the working area of the tested magnet in either $x$ or $y$ direction with the return wire held fixed either inside the bore of the magnet or external to the magnet.

There are two main modes of operation depending on how the flux change seen by the stretched wire is created:

- Measurements with DC magnet current, when the wire is moving to change the flux. This mode supposes a relatively high field and therefore is applicable mostly to the cold test.

- Measurements with AC magnet current, when the wire is stationary placed on a few preselected positions inside the working area of the magnet, reads the flux change due to the AC excitation. This mode is more sensitive than DC and therefore applicable mostly to the warm test.

The main features of the SSW system are the following:

- stage units with $150 \mathrm{~mm}$ travel, $1 \mu \mathrm{m}$ linear encoders, 25 $\mu$ rad orthogonality;

- precise wire support on ceramic ball-bearings referenced within $10 \mu \mathrm{m}$ to a laser tracker retro-reflector targets;

- tilt sensor with $10 \mu \mathrm{rad}$ resolution; 
- sag removal using tension measurement, based either on a gauge or on a wire vibration frequency;

- counter-directional stage motions: "true axis" in addition to average axis (see below);

- measurements with AC (warm measurements) or DC magnet current.

The above-mentioned counter-directional motion-"true axis", when the two stages are moving in opposite direction but in the same plane extends the ability of the system. This operational mode offers not only the average strength ( $\left.\int \mathrm{Gdl}\right)$, axis $(\mathrm{x}, \mathrm{y})$ and field direction $\left(\int \alpha \mathrm{dl}\right)$, but also the first moment of the axis (average pitch and yaw).

2) System Performance: Two special tests to qualify the system were executed on SSS5 at warm temperature.

Random errors - The derived repeatability of two runs of measurements performed with the system reinstallation between runs is as follows:

- position of magnetic axis within $60 \mu \mathrm{m}$;

- inclination of magnetic axis within $20 \mu \mathrm{rad}$;

- roll angle within $0.13 \mathrm{mrad}$.

Systematic errors-roll angle system offset-The absolute accuracy of the roll angle measurement was derived from a test performed swapping the stages while leaving the magnet stable. The system roll offset obtained is 0.11 mrad with $0.06 \mathrm{mrad}$ of uncertainty. Note that this offset measured before shipping the system to CERN was 0.187 mrad. We are looking at present on the reason for this drift, that may imply periodical calibration. We remark however that the scattering of roll angle derived from this measurement is safely within requirements.

It was discovered during all those tests that the SSW system is significantly sensitive to the following factors:

- position of the return wire-especially at AC excitation, caused by the stray field;

- environmental conditions such as mechanical vibrations, temperature profile inside the anti-cryostat, humidity etc;

- magnetic susceptibility of the wire, as it was observed on the recently purchased spool of the $\mathrm{CuBe}$ wire that it is sensitive to the magnetic field.

It implies that prior to perform correctly any cross-calibration between the systems one has to investigate all those effects.

\section{Comparison Between the Two Systems}

The absolute accuracy of the axis measurement was derived from a test performed on SSS5 at room temperature with the two considered systems: the SSW and the Scanner. The comparison between the systems gives the following differences:

- lateral position of magnetic axis within $0.2 \mathrm{~mm}$;

- inclination of magnetic axis in yaw within $0.02 \mathrm{mrad}$;

- inclination of magnetic axis in pitch within $0.035 \mathrm{mrad}$.

This can be attributed either to the systematic error of one of the system or to the different measurement conditions$20 \mathrm{~A}$ DC in case of the automated scanner and $5 \mathrm{~A}$ AC in case of SSW.

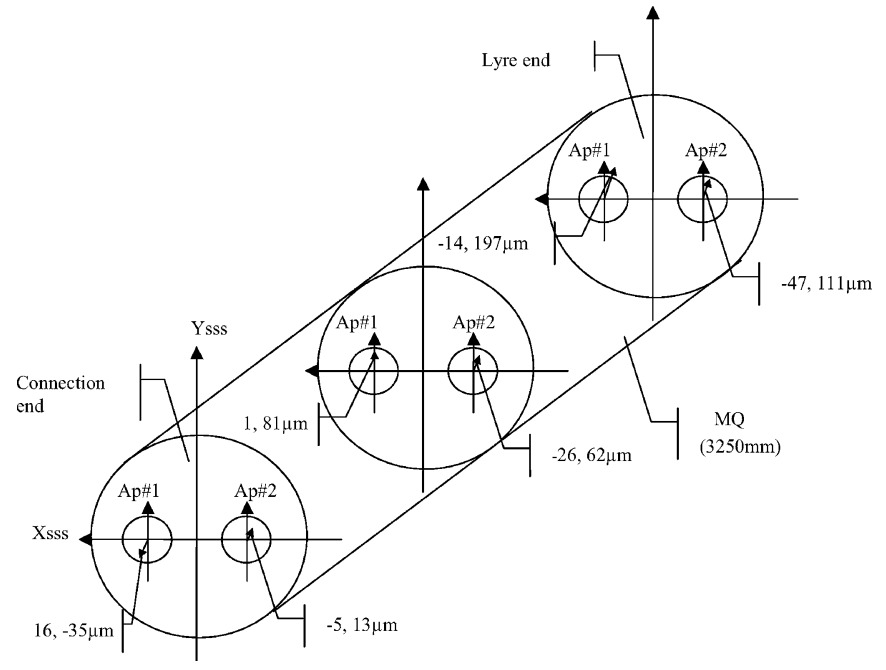

Fig. 1. The movement of SSS5 magnetic axis due to the transportation-each vector of movement is presented by two components: $\mathrm{x}$ and $\mathrm{y}$ in $\mathrm{mum}$.

\section{Study OF SSS MAGNetic AXIS Stability}

\section{A. Transportation Influence}

This test was conducted with SSW in warm condition only. The measurements were executed twice before and after the transportation to Germany and back to CERN-in total $1500 \mathrm{~km}$. The movements of the magnet caused by the transportation are illustrated in Fig. 1 and are as follows:

\section{Magnetic axis (midpoint)}

- Vertical: the magnet midpoint moved upward with respect to the fiducials by $80 \mu \mathrm{m}$ and $60 \mu \mathrm{m}$ for apertures 1 and 2 respectively;

- Horizontal: the magnet midpoint was moved sideways with respect to the fiducials by $0 \mu \mathrm{m}$ and $-25 \mu \mathrm{m}$ for apertures 1 and 2 respectively.

Yaw: the magnet was turned in horizontal plane by $-10 \mu \mathrm{rad}$ for both apertures. This is equivalent to the movement of the lyre end of the MQ by $-15 \mu \mathrm{m}$ for aperture 1 and $-50 \mu \mathrm{m}$ for aperture 2 and for the connection end by $15 \mu \mathrm{m}$ and by $-5 \mu \mathrm{m}$ respectively. Note that all those values are within the scattering of measurement.

Pitch: the magnet was turned in vertical plane by $-70 \mu \mathrm{rad}$ and by $-30 \mu \mathrm{rad}$ for aperture 1 and 2 respectively. This is equivalent to the movement of the lyre end of the MQ by $197 \mu \mathrm{m}$ and $110 \mu \mathrm{m}$ and for connection end: $-35 \mu \mathrm{m}$ and $15 \mu \mathrm{m}$ for apertures 1 and 2 respectively (see Fig. 1). The observed movement of the MQ in pitch exceeds the random error and therefore has being reliably identified.

Roll angle: the difference in roll angle is $0.054 \mathrm{mrad}$, which is inside random spread of measurements.

Distance between aperture axes: the difference in the inter-aperture distance obtained from two campaigns of measurements is less than $0.027 \mathrm{~mm}$, which is inside the accuracy window.

\section{B. Movement of the Cold Mass During Cooling Down}

The cold mass is positioned inside the cryostat on two GFRE support posts, one of them is fixed on both ends while the second 


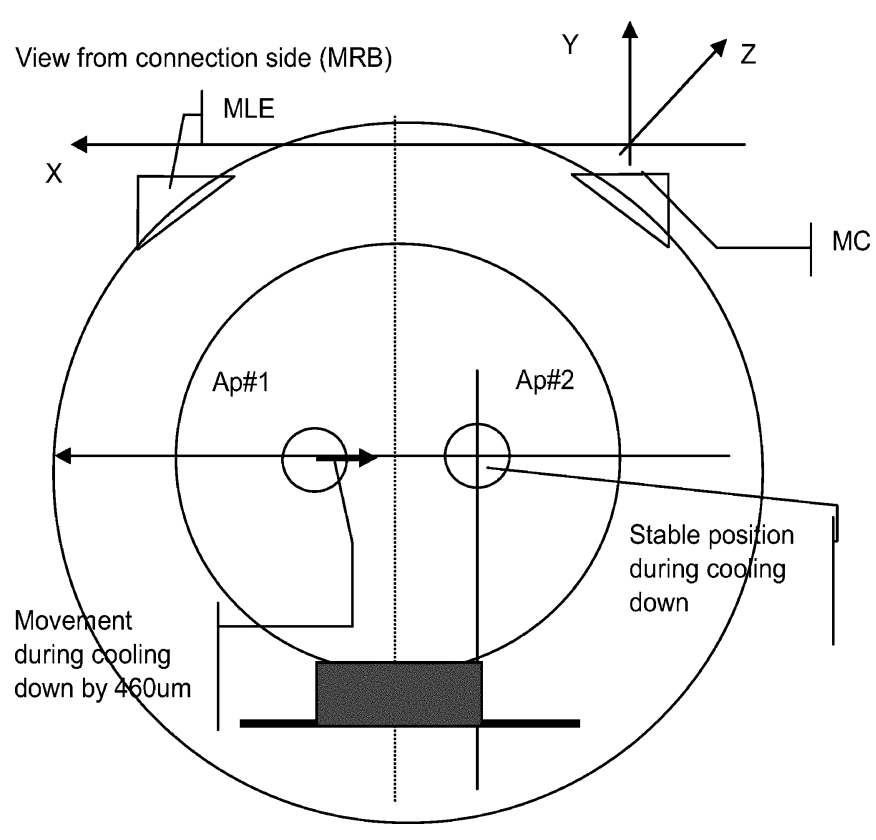

Fig. 2. Lateral movement of cold mass midpoint during cooling down.

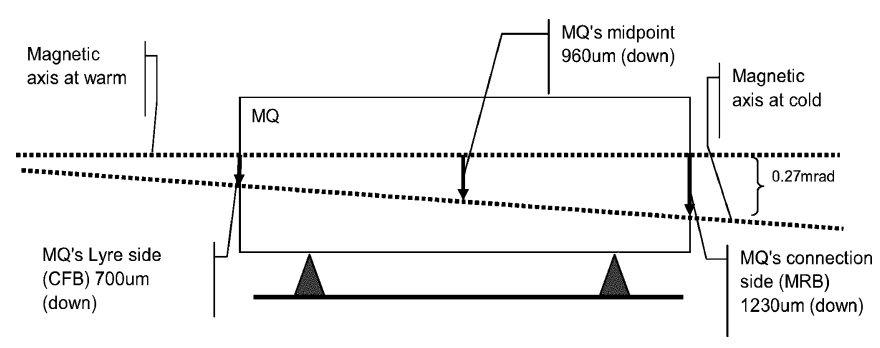

Fig. 3. Vertical movement of the cold mass during cooling down.

one can axially slide. This test was executed with the SSW measuring the axes at warm and then at cold. The results obtained are illustrated in Figs. 2 and 3 and summarized in Table I.

1) Movement in Horizontal Plane: As one can see from Fig. 2 the lateral movement of the cold mass was not symmetric with respect to the geometric axis of the magnet i.e., the position of aperture 2 was stable during the cooling, while almost whole lateral contraction was observed in aperture 1 . The obtained total contraction of MQ midpoint is in good agreement with prediction $-460 \mu \mathrm{m}$. It means that in addition to contraction the whole magnet moved by approximately $230 \mu \mathrm{m}$ to the right with respect to its geometrical axis. As one can see from the Table I the inclination in yaw is rather negligible $-0.03 \mathrm{~mm}$ and is within the tolerance.

2) Movement in Vertical Plane: Even if the predicted contraction in vertical direction of approximately $1 \mathrm{~mm}$ is perfectly matched to what was measured at the midpoint of both MQ (see Fig. 3), the two ends of the MQ's moved differently, namely by $0.7 \mathrm{~mm}$ on the Cryogenic Feeding Box (CFB) side meanwhile on Magnet Return Box (MRB) side movement observed is $1.23 \mathrm{~mm}$ - both downwards.

There are few probable physical models explaining this phenomenon:
TABLE I

SuMMARY OF THE MAGNETIC AXIS POSITION OF SSS5

\begin{tabular}{lrrr}
\hline \hline Centering & Transport effect & Cooling effect & Thermocycle effect \\
Parameters & & & \\
X_\#1[mm] & 0.005 & 0.463 & -0.017 \\
Yaw\#1[mrad] & -0.013 & 0.012 & -0.008 \\
Y_\#1[mm] & 0.095 & 0.988 & \\
Pitch\#1[mrad] & 0.063 & -0.251 & \\
X_\#2[mrad] & -0.017 & 0.002 & -0.015 \\
Yaw\#2[mrad] & -0.015 & 0.010 & \\
Y_\#2[mm] & 0.056 & 0.928 & 0.045 \\
Pitch\#2[mrad] & 0.031 & -0.292 & \\
Interaperture & 0.022 & 0.461 & -0.002 \\
\hline \hline
\end{tabular}

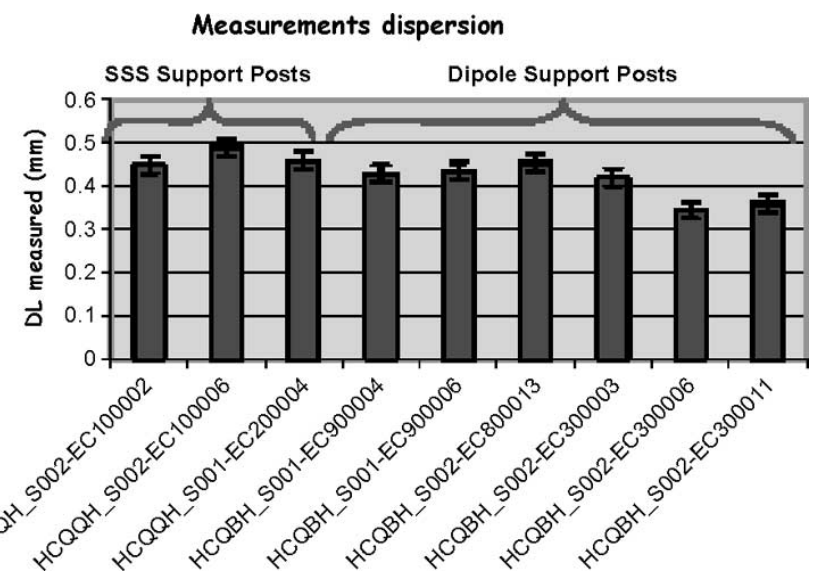

Fig. 4. Measurements dispersion of the SSS support posts.

- The bellows connecting the SSS to the Cryogenic Feed Box are not flexible enough - during cooling they apply a vertical force to the cold mass slightly lifting it;

- Contraction of the support posts is not the same due to their different temperature conditions;

- Axial forces occurring during cooling could redistribute the load between the feet unequally, which can also lead to inclination of the cold mass in pith;

- The magnet moved inside the inertia tube.

A special test of the SSS support posts contraction has been done in liquid nitrogen. The results of these test are illustrated in Fig. 4. Based on the measurements, the calculation estimates to $0.31 \mathrm{~mm}+/-0.02 \mathrm{~mm}$ the thermal contraction in LHC operating conditions.

All aforementioned models need to be further investigated in more detail, although the requirement to the pitch of quadrupole is quite soft and the observed amount of tilt is safely acceptable for the LHC machine.

\section{Effect of Thermal Cycle}

This test was done by measuring twice the position of the magnetic axes with the SSW at cold with thermal cycle in between. The maximal derived difference is of the order of $45 \mu \mathrm{m}$ and is within the SSW system resolution window (Table I). 


\section{CONCLUSIONS AND OUTLOOK}

\section{A. Magnet Performance}

The results of extensive investigations of the magnetic axis behavior carried out on the SSS prototype (SSS5) can be summarized as follows:

- The effect of transportation of the SSS from the vendor site to the CERN is visible however the measured displacements are within the tolerance window.

Moreover, because all magnets should be tested at cold before their installation - this instability is not an important issue. More critical is the stability of axes after the cold tests are done.

- The contraction of the magnet position during the cooling down is remarkably different with respect to what was expected (horizontal displacement of $0.23 \mathrm{~mm}$ ). In vertical plane a movement of the order of $0.3 \mathrm{mrad}$ of inclination in pitch was found. Despite the fact that it is not very critical for the machine performance this effect should be further investigated.

- The thermal cycle effect is found to be weak and is within the measurement system resolution.

Based on results obtained for SSS5, it is evident that to establish a reliable cold-warm correlation the proper statistics is still needed, which we will gather from the first series SSS.

\section{B. Measurement Systems Performance}

The mutual cross-calibration between the two main measurement systems revealed a maximum difference of $0.2 \mathrm{~mm}$. Even if it might be interpreted as a systematic error, this small discrepancy could be caused by different measurement conditions needed for those systems. The last requires further investigations.

\section{ACKNOWLEDGMENT}

The authors would like to thank all MTM staff involved in these rather delicate measurements and the tests preparation and execution. Many thanks also for very fruitful discussions to L. Bottura, A. Siemko and B. Skoczen.

\section{REFERENCES}

[1] T. Tortschanoff et al., "The short straight sections for the LHC," in Proc. PAC-1997, pp. 3374-3376.

[2] J. DiMarco, H. Glass, M. J. Lamm, P. Schlabach, C. Sylvester, J. C. Tompkins, and J. Krzywinski, "Field alignment of quadrupole magnets for the LHC interaction regions," IEEE Transactions on Applied Superconductivity, vol. 10, no. 1, pp. 127-130, March 2000.

[3] L. Bottura, F. Chiusano, O. Dunkel, P. Legrand, S. Schloss, P. Schnizer, P. Sievers, and N. Smirnov, "A system for series magnetic measurements of the LHC main quadrupoles," IEEE Transactions on Applied Superconductivity, vol. 12, no. 1, pp. 168-169, March 2002 